a method of directly monitoring cerebral activity and oxygenation during transition and resuscitation after birth.

Methods Neonates $>34$ weeks gestation born via caesarean section were included. Cerebral activity was continuously measured with amplitude integrated EEG (aEEG) and cerebral oxygenation (rSO2) with near-infrared-spectroscopy during the first ten minutes after birth. For quantitative analysis of aEEG the mean minimum amplitude (Vmin) and maximum amplitude (Vmax) was determined at every minute. Neonates with normal transition were compared to neonates with need of resuscitation.

Results Out of 224 eligible neonates 63 were included and 46 had reliable measurements: 31 with normal transition and 15 in need of resuscitation. Neonates with normal transition showed higher values for $\mathrm{Vmin}$ in the third minute and higher values for Vmax in the third and fourth minute compared to minute 10. Neonates requiring respiratory support had lower values for $\mathrm{Vmin}$ in the ninth minute compared to minute 10 . In neonates with normal transition $\mathrm{rSO} 2$ values during the first six minutes were lower when compared to minute 10 . rSO2 values in neonates requiring respiratory support remained lower over the first eight minutes when compared to minute 10 .

Conclusions This is the first study demonstrating the feasibility of aEEG and rSO2 monitoring during neonatal transition. The cerebral activity pattern in compromised infants requiring resuscitation was different when compared to infants with normal transition.

\section{EARLY PREDICTION OF OUTCOME IN EXTREMELY PRETERM INFANTS USING CRANIAL ULTRASOUND AND MRI SCORING SYSTEMS}

doi:10.1136/archdischild-2012-302724.0225

1,2B Skiöld, 'B Vollmer, ${ }^{2,3} \mathrm{~B}$ Hallberg, 1,2U Ådén, ${ }^{2,3} \mathrm{M}$ Blennow, ${ }^{3,4} \mathrm{~S}$ Horsch. 'Dept of Women's and Children's Health, Karolinska Institutet; ${ }^{2}$ Dept of Neonatology, Karolinska University Hospital; ${ }^{3}$ Dept CLINTEC, Karolinska Institutet, Stockholm, Sweden; ${ }^{4} D e p t$ of Neonatology, Erasmus MC - Sophia Children's Hospital, Rotterdam, The Netherlands

Background and aims Extremely preterm infants are at high risk of brain injury and adverse outcome. This study compares the predictive values of cranial ultrasound (CUS) and conventional magnetic resonance imaging (MRI) at term equivalent age (TEA) for cerebral palsy $(\mathrm{CP})$ and/or severe cognitive delay.

Methods Eighty-four infants, born $<27$ weeks of gestation, underwent MRI (1.5 T) and CUS (5-8 MHz) at TEA. Images were evaluated by three independent observers. A previously published (Horsch et al. 2010), modified CUS scoring system and a widely used MRI scoring system were applied (Woodward et al. 2006). At age 30 months corrected, 62 infants (74\%) underwent neurological examination and assessment with the Bayley Scales of Infant and Toddler Development-III (BSITD-III). Cut off levels for developmental delay ( $-2 \mathrm{SD})$ were adjusted to an age and sex matched term born control group $(\mathrm{n}=85)$.

Results Both CUS and MRI scoring systems predicted CP with a sensitivity of $75 \%$, while specificity was higher for MRI $(96 \%$, versus $91 \%$ ). Predictive values for severe cognitive delay were equal and good, with sensitivities of $100 \%$ (specificity $95 \%$ for MRI, $90 \%$ for CUS).

Conclusion We conclude that, using the scoring systems applied in this study, both CUS and MRI at TEA are useful in predicting CP and/or severe cognitive delay at age 30 months. Furthermore, we suggest that these scoring systems, which can be used in clinical routine, are helpful tools in identifying infants who are at high risk of adverse outcome and should undergo MRI.

\section{NUTRITION IN THE CRITICALLY ILL: ENTERAL AND PARENTERAL NUTRITION IN THE NEWBORN}

doi:10.1136/archdischild-2012-302724.0226
${ }^{1} \mathrm{C}$ Agostoni, ${ }^{2} \mathrm{G}$ Francescato, ${ }^{2} \mathrm{M}$ Agosti. ${ }^{1}$ Pediatric Department 2, Fondazione IRCCS Policlinico, University of Milan, Milan; ${ }^{2}$ NICU, Del Ponte Hospital, Varese, Italy

Initiating both parenteral nutrition with adequate protein:energy ratios and early enteral feedings strategies (within the first hours of life, whenever possible) seem to offer preventive advantage towards developing complicating illnesses and favour neurodevelopment. Human milk as early enteral feeding has trophic effects on the gastrointestinal tract and shows anti-infectious properties. The requirements of growth and organ development create a challenge in nutritional management of newborn infants, especially preterms and those with additional disorders, such as intestinalfailure, bronchopulmonary dysplasia, and sepsis. Nutritional support improves outcome in critically ill infants but is affected by fluid restriction, metabolic derangements, gastric intolerance and feeding interruptions. Few studies compare more critically ill infants with less critically ill infants as far as nutritional support during the initial weeks of life looking at growth and rates of adverse outcomes. Accordingly, the influence of critical illness on the risk of adverse outcomes seems to be mediated by energy intakes during the first week of life (Pediatr Res 2011; 69:522). Changes in amounts and ratios of protein and energy, fat quality (medium chain triglycerides, oleic acid and n-3 long-chain polyunsaturated fatty acids through parenteral nutrition), maintaining normoglycemia during full or partial parenteral nutrition, shortand medium term effects of either parenteral or enteral glutamine supplementations, daily supplemental zinc, rate of feeding advancements and avoidance of postnatal growth retardation represent the main items whose roles in critically ill preterm infants have been considered so far. Available data are still limited and do not allow for firm conclusions in most cases.

\section{AMOUNT AND TIMING OF OWN MOTHERS MILK INTAKE IS ASSOCIATED WITH THE RISK OF NEC, SEPSIS OR DEATH IN PRETERM INFANTS}

doi:10.1136/archdischild-2012-302724.0227

${ }^{1}$ W Corpeleijn, 'S Kouwenhoven, ${ }^{2} \mathrm{M}$ Paap, ${ }^{1} \mid$ van Vliet, ${ }^{3}$ Scheerder, ${ }^{3} Y$ Muizer, ${ }^{3} \mathrm{O}$ Helder, ${ }^{1,23} \mathrm{H}$ van Goudoever. 'Pediatrics, VU University Medical Center; ${ }^{2}$ Pediatrics, Amsterdam Medical Center, Amsterdam; ${ }^{3}$ Pediatrics, Erasmus MC - Sophia Children's Hospital, Rotterdam, The Netherlands

Background Necrotising enterocolitis (NEC) incidence is lower in preterm infants who are fed their own mother's milk (OMM) compared to formula. Possible mechanisms include protective factors of human milk or detrimental effects of formula. OMM is often unavailable, especially in the first few days following birth, while frequently donor milk is not available either.

Objectives To quantify the association between OMM intake during the first days of life and the incidence of sepsis, NEC and death.

Methods Retrospective single centre study in infants with a birth weight $<1500 \mathrm{~g}$. Intake of OMM/formula during the first ten days of life were recorded. Episodes of sepsis, NEC and death were registered during 60 days. Statistics: Cox regression analysis.

Results $\mathrm{n}=349$ infants. OMM intake during the first 5 days of life was associated with a lower incidence of NEC, sepsis and/ or death during the first 60 days of life (hazard ratio(HR) of the category $0.01-50 \%$ OMM intake: 0.49 [95\%CI 0.28 , 0.87], HR of the category $50.01-100 \%$ OMM intake: 0.50 [95\% CI 0.31 , 0.83 , compared to no OMM). During days 6-10, the protective effect was present if $>50 \%$ of intake was OMM ( $\mathrm{HR}=0.37$ [95\%CI 0.22, 0.65]).

Conclusion Any human milk during the first 5 days of life is associated with an decreased risk of $\mathrm{NEC/sepsis/death,} \mathrm{while} \mathrm{the}$ amount of human milk seems important from day $5-10$. 